\title{
A Wide Range and High Swing Charge Pump for Phase Locked Loop in Phasor Measurement Unit
}

\author{
Motahhareh Estebsari \\ Department of Electronics and Telecommunications \\ Politecnico di Torino \\ Turin, Italy \\ motahhareh.estebsari@polito.it
}

\author{
Abouzar Estebsari \\ Department of Energy \\ Politecnico di Torino \\ Turin, Italy \\ abouzar.estebsari@polito.it
}

\begin{abstract}
Phasor Measurement Units are widely utilized in power systems to provide synchrophasor data for a verity of applications, mainly performed by Energy Management Systems (EMS). Synchrophasors are measured at different parts of the network and transmitted to Phasor Data Concentrator (PDC) at a rate of 30-60 samples per second. The synchronization is done by means of a phase locked oscillator inside PMU which uses clock signal of the Global Positioning System (GPS). In this paper a novel charge pump with an appropriate operation capability in phaselocked-loops is presented. By using this phase locked loop in phase measurement unit, the total performance of this circuit will be improved. The proposed charge pump uses current mirror techniques in order to achieve a wide range of output voltage to control the oscillator and also has a good performance in a wide range of frequency from $33 \mathrm{MHz}$ to 555MHz. This circuit is designed and simulated in TSMC 0.18um CMOS technology. The proposed charge pump only consumes $390 \mathrm{uW}$ power in supply voltage of $1.8 \mathrm{~V}$ at $500 \mathrm{MHz}$ and has a maximum current of $16.43 \mathrm{uA}$ with a acceptable current matching between source and sink currents. It is also capable to be used in a wide frequency range and low power applications.
\end{abstract}

Keywords - charge pump, phase locked loop, phasor measurement unit, low power.

\section{INTRODUCTION}

Phasor Measurement Units (PMUs) play an important role in power system wide area monitoring supporting dynamic operation and control of system. Critical parameters of power system like voltage of nodes, current of lines, load angle, and system frequency should be monitored in real time to avoid system failures in case of fault occurrence [1]. For proper monitoring, synchronized measurement of power system parameters is needed. PMUs provide such synchronized time-stamped data using global positioning system (GPS) clock. The data from PMUs are widely used in frequency control systems [2], state estimation [3], power quality measurement [4], etc. PMU sends the synchro-phasor data to the phasor data concentrator (PDC) through unicast or multicast. A block diagram of key components of a PMU is shown in Fig. 1. Current and voltage transformers (CT and VT) collect current and voltage measurements from the field. These signals are sent to analog to digital converts through an antialiasing filter which limits the bandwidth of instantaneous signal based on sampling theorem. The analog to digital converter works according to IEEE C37.118 standard specifications. The digital output will be then converted to phasors by a phasor estimator. The GPS clock uses a crystal oscillator to provide clock pulses. The possible errors between pulses per second and clock frequency is then corrected by a phase locked oscillator. The outcome of the overall system is synchro-phasor data to be communicated to the PDCs [5].

Phase locked oscillator utilizes phase locked loop (PLL). PLL is widely used in integrated circuits like time recovery, frequency multipliers and synthesizers [6], timing extraction from data streams, memories, hard disk drive electronics, clock generation, microprocessors, clock and data recovery, and in phase measurement unit (PMU). A PLL with a desired performance has short settling time, and generates low noise and low spur signals. Based on their applications, PLL can be classified into digital PLLs, programmable PLLs, PLL-based frequency synthesizers, etc. Moreover, according to the type of phase detector used in PLLs, they can be divided into "simple" and "charge pump" PLLs (CPPLL); where the former has simple phase detector, and the latter uses phase frequency detector coupled with charge pump. Looking closely to the structure of CPPLLs, one can find a basic PLL which is extended with a charge pump put between phase detector and loop filter. Charge pump is used to inject and remove current to/from loop filter for integration operation [7]. The challenges during the charge pump design include input and output currents (UP and DN currents), low power consumption, operation frequency range and circuit output voltage range [8].

As mentioned, one of the applications of charge pump circuit is in phase locked loops (PLLs). An analog PLL circuit according to Fig. 2 consists of important blocks of phase frequency detector (PFD), charge pump (CP), loop filter (LF) and voltage-controlled oscillator (VCO) [9], so one of the main difference between phase locked loop and delay locked loop (DLL) is that delay locked loop uses voltage controlled delay line instead of VCO [10-11]. In an analog PLL, a phase frequency detector, receives its inputs from reference input signal and the output of VCO and its output is based on phase difference between its inputs. In fact, this output determines charge pump operation procedure and the output of PFD is transmitted to charge pump circuit as a voltage pulse or current pulse. 
Finally, loop filter, integrates charge pump output. In order to obtain the desired frequency of output signal, the voltage control oscillator is regulated by the capacitor voltage. Loop filter, upon triggered to operate, and minimizes the phase difference among phase detector inputs by setting the appropriate control voltage. When frequency difference is small enough, the mechanism of PLL feedback makes the input frequencies of the 2 phase detectors equal. At this time, VCO is actually locked with input frequency. This is a locked state of PLL.

In Fig. 3, structure of a conventional charge pump is depicted. It uses negative OPAMP feedback technique and has good time of switching and perfect current matching. It uses body bias to prevent the current increase [12].

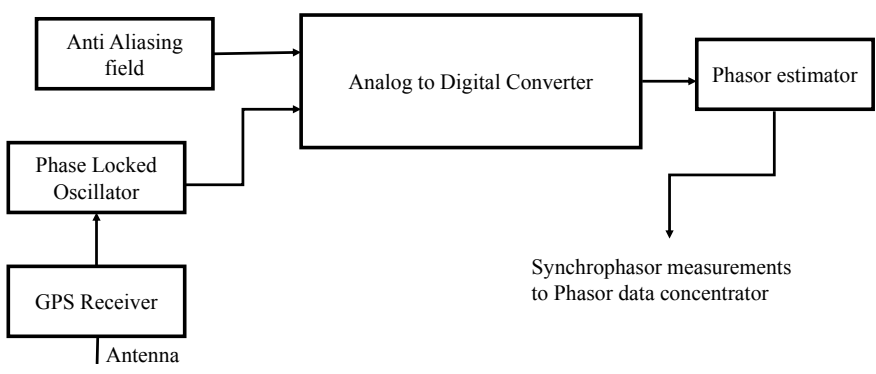

Fig. 1. Block diagram of Phasor Measurement Unit (PMU)

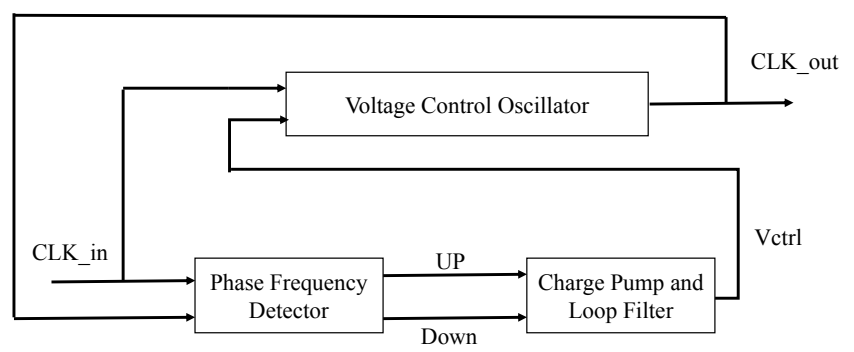

Fig. 2. Block diagram of charge pump based phase locked loop (CPPLL)

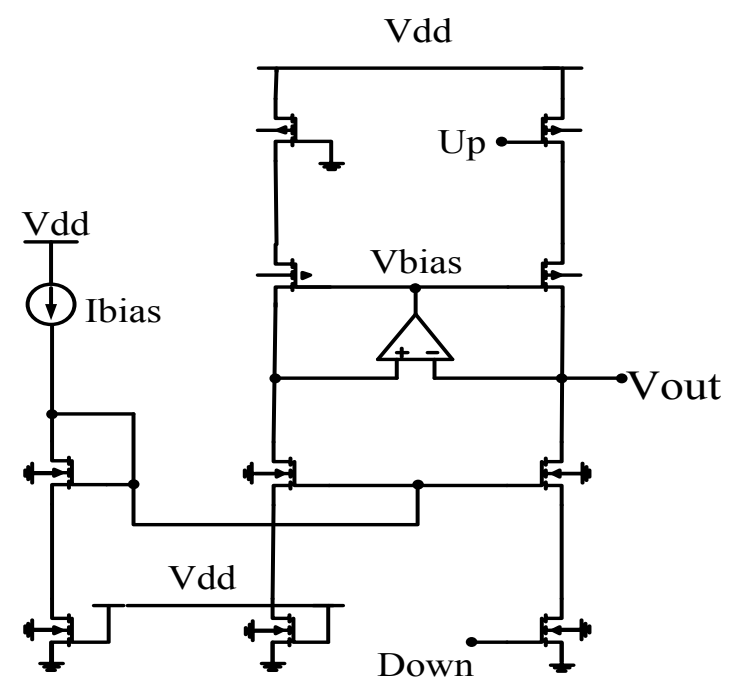

Fig. 3. Structures of a conventional charge pump
The channel length modulation effect is reduced by using negative feedback, but this CP has a high-power consumption and area. To improve these problems, the structure in [13] is presented. This charge pump has a high UP and DOWN currents and the current variation is not very low in all range of output voltage of $\mathrm{CP}$. The other structure which is used as charge pump, and does not this problem, utilizes adaptive body bias to compensation current variation which results to good current matching [14]. This charge pump has low power consumption, but when charge pump needs to be protected from switch noises, this topology may have some concerns. Moreover, this structure has a high UP and DOWN currents. An improvement for this type of charge pump is presented in [15], which uses rail-to-rail input stage and have four current sources, two regulation loops, and three OPAMPs to reduce current mismatch and to use for short-channel devices. But, this CP has high source current and sink current.

The rest of this paper is structured as follow: in section II the proposed charge pump is introduced. In section III the simulation results of the proposed circuit is discussed and at the end in section IV a conclusion is provided.

\section{PROPOSED CHARGE PUMP}

Charge pump is one of the important blocks in delay locked loops and phase locked loops which aim to control this circuits operation and performance. Structure of the proposed charge pump is shown in Fig. 4.

It consists of a differential amplifier which has NMOS transistors of M3 and M4, and they are placed in the center of this CP. The outputs of this transistors affects in the M8 and M9 and therefore, when Down signal is high, M3 is on and M4 is low, so the current of M6 is injected to the M3. By the current mirror sources consists of M10 and M7 the Down current can affect the loop filter. In fact, with the proper relation between the gate width of the current mirror (M10 and M7), the suitable Down current is produced and discharge the capacitor in loop filter.

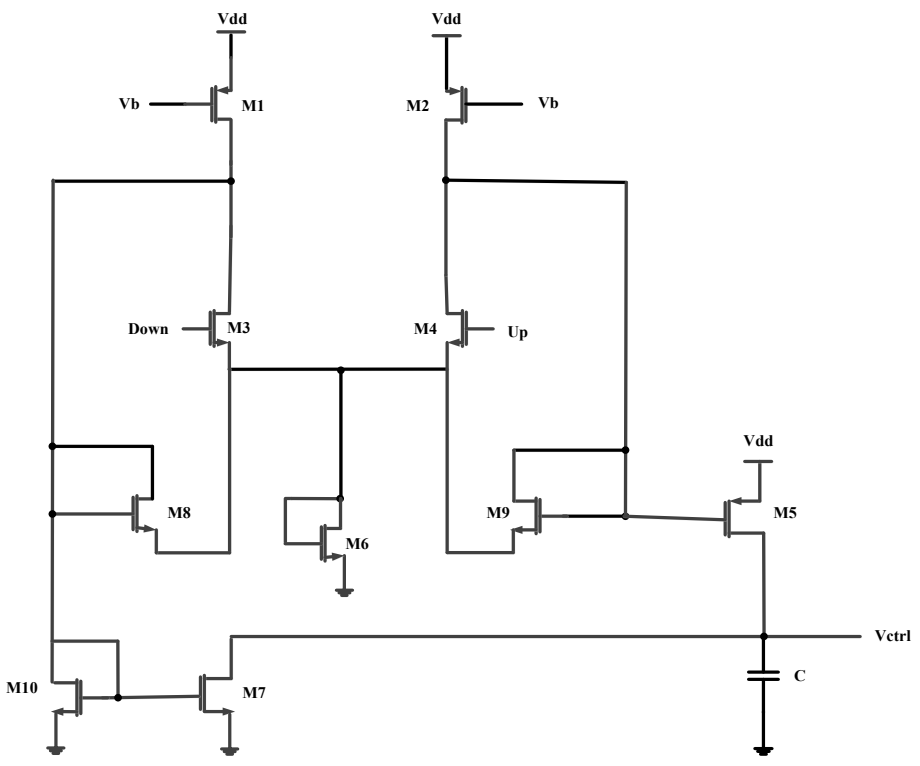

Fig.4. The proposed charge pump 


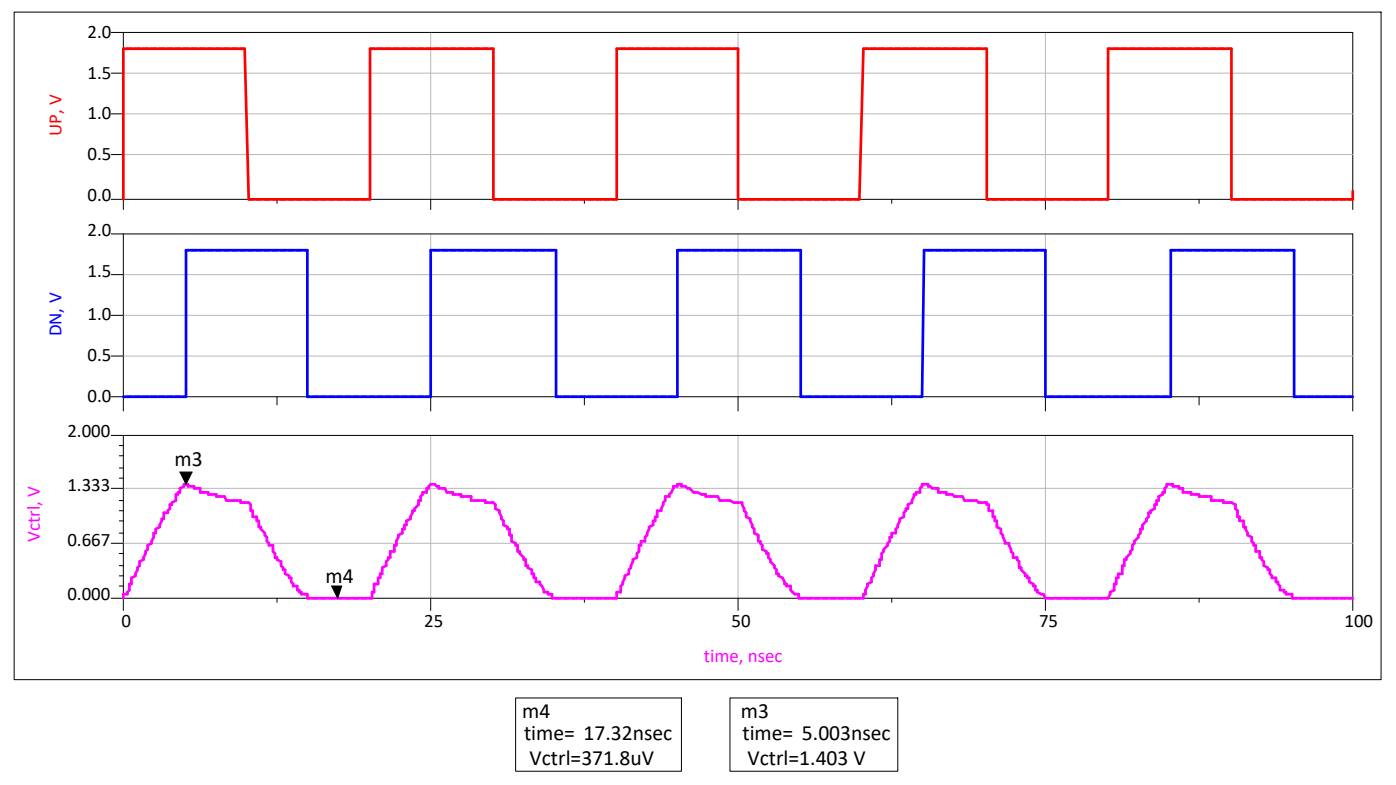

(a)

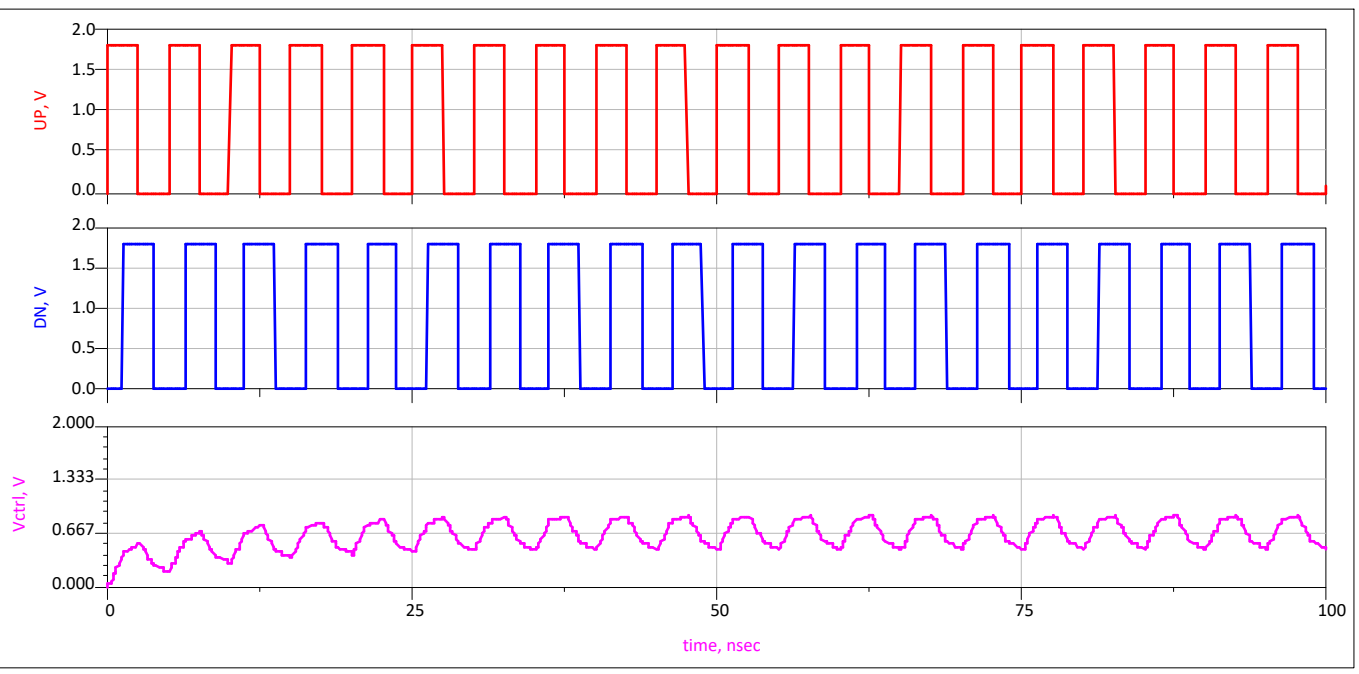

(b)

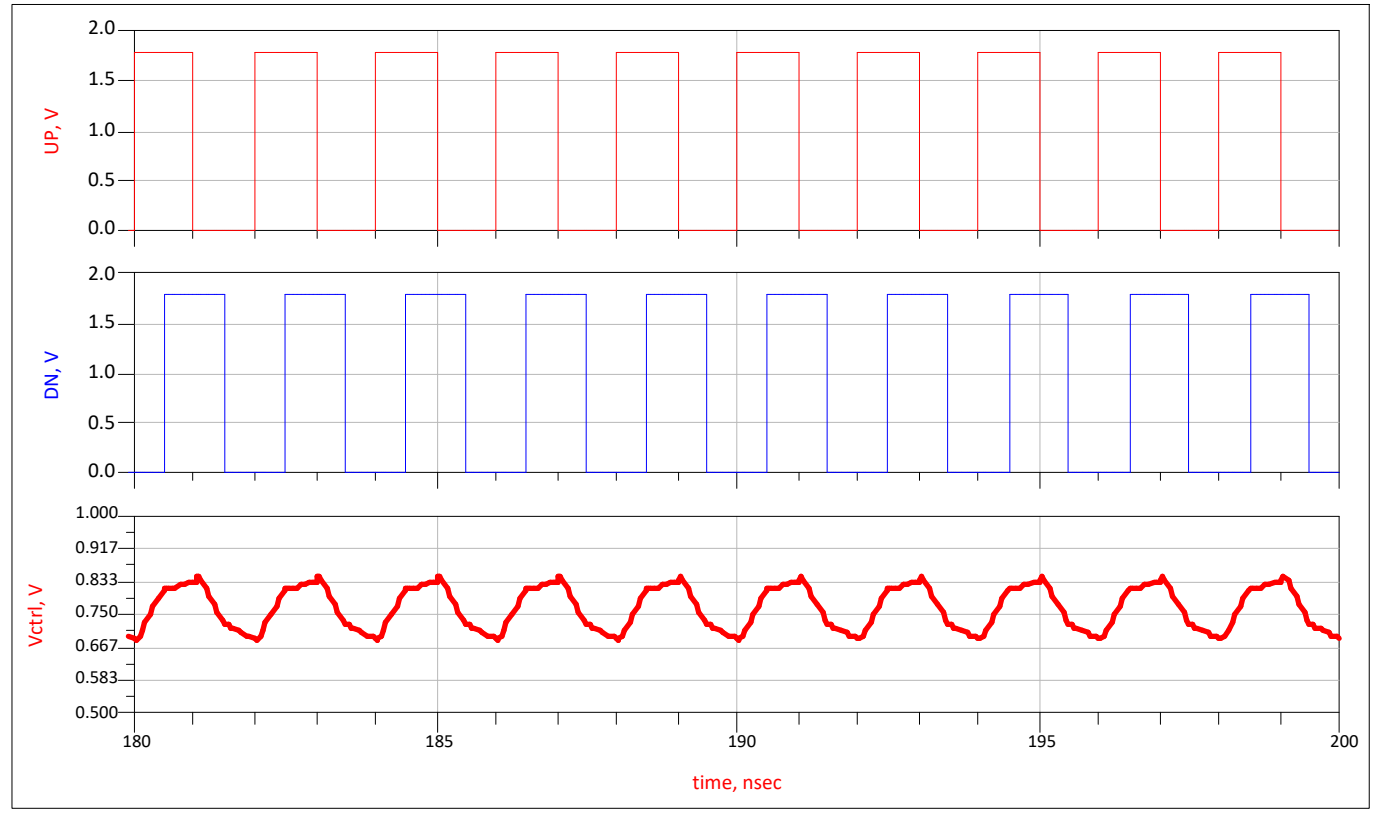

(c)

Fig. 5. UP, DN, and Vctrl (output of charge pump) signals at: a) $50 \mathrm{MHz}$, b) $200 \mathrm{MHz}$, c) $500 \mathrm{MHz}$ 
Transistors M2 and M1 are biased by a voltage source of $0.8 \mathrm{~V}$. On the contrary, when UP signal is high, the current of M6 is injected to the M4, so M4 and M9 will be on and with the transistor of M5 the current will inject to the capacitor. So in this case, the capacitor will charge. This would result in accuracy and also increase of control voltage range. The NMOS differential pair (M3 and M4) which is considered in the center of circuit activates the current mirror sources according to the Up and DOWN input signals. To charge the capacitor of loop filter, the proposed charge pump uses the direction consist of transistors M4, M9 and M5. Considering the correlation between input and output currents to and from loop filter, this is easily observed by using the simulation tool and through DC analyses. In other words, the output current from M5 is equal to the current from M7. Therefore, in this case charge pump carries out charge and discharge operation of output node capacitance with the same speed.

\section{SIMULATION AND RESULTS}

The proposed charge pump circuit is designed and simulated with TSMC 0.18um CMOS technology. A challenge in increasing the output voltage of charge pumps is the need to increase the value of current source. Since current increase will result in bigger power consumption of the device, the output voltage swing of charge pumps is hence limited. But as it is shown in Fig. 5 (a), in this proposed charge pump at $50 \mathrm{MHz}$, the result for voltage of charge pump (CP), shows range of $0 \mathrm{~V}$ to $1.403 \mathrm{~V}$ that is appropriate and also the power consumption will be low. Moreover, this voltage range is suitable to control voltage controlled oscillators which operate in a phase locked loop. In Fig. 5 (b and c) the waveform of output control voltage are depicted for frequencies $200 \mathrm{MHz}$ and $500 \mathrm{MHz}$. In Fig. 6 , Up and Down current curves are shown with respect to control voltage. This charge pump generates a suitable current matching with maximum current of $16.43 \mathrm{uA}$. As shown in Fig.5, when the control voltage (Vctrl) is from $0.8 \mathrm{~V}$ to $1.4 \mathrm{~V}$, the charge pump will have an acceptable current variation. In addition, the proposed circuit consumes $315.8 \mathrm{uW}$ power at $50 \mathrm{MHz}$ with supply voltage of $1.8 \mathrm{~V}$. Also, it can be used in an analog PLL.

In Fig. 7, the amount of up and down currents (I_UP and I_DN) are shown in process, voltage and temperature variations (PVT), when control voltage changes from 0 to Vdd. For example, at fast- fast and slow- slow condition, the maximum currents are $29 \mathrm{uA}$ and $9.3 \mathrm{uA}$, respectively.

As it was discussed earlier, this charge pump has a very good performance in frequency range of $33 \mathrm{MHz}$ to $555 \mathrm{MHz}$ with a power consumption range of $308 \mathrm{uW}$ to $387 \mathrm{uW}$ for $33 \mathrm{MHz}$ and $555 \mathrm{MHz}$, respectively. Power consumption of this CP in PVT variations is summarized in TABLE I, and in TABLE II a comparison between previous charge pump structures with this proposed structure is provided.

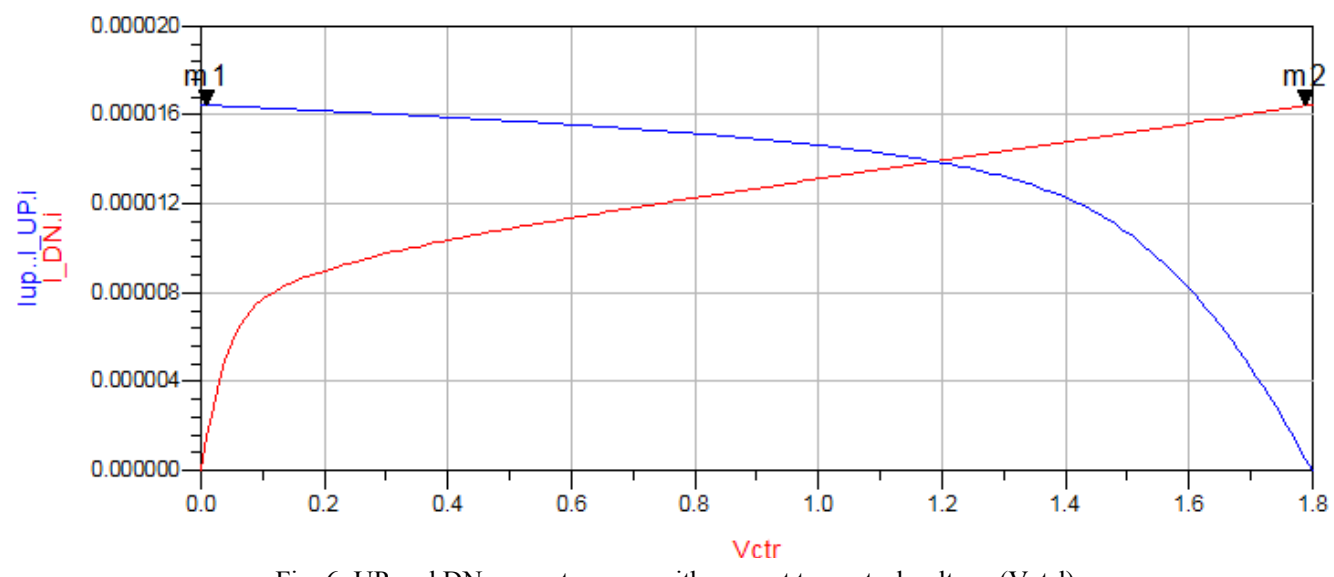

Fig. 6. UP and DN current curves with respect to control voltage (Vctrl)

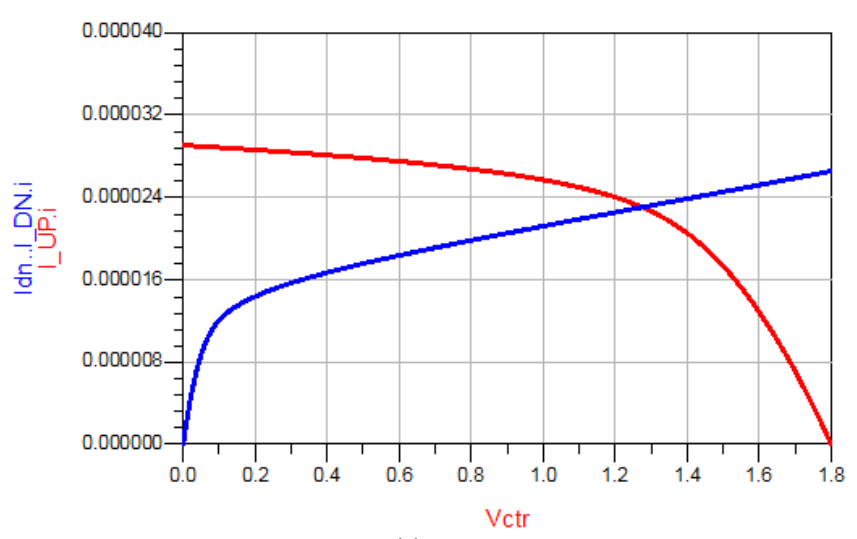

(a)

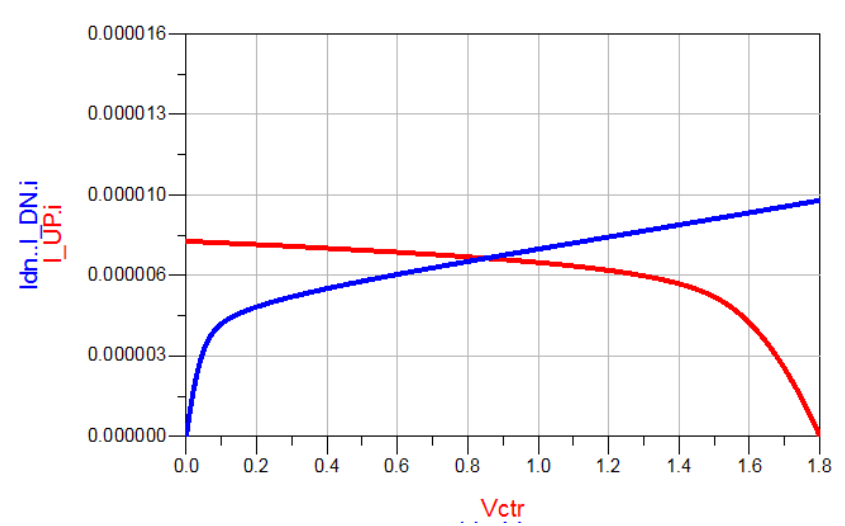

(b)

Fig. 7. The amount of Up and DN currents when the voltage control changes from 0 to Vdd for corner cases of, a) Fast Fast, b) Slow Slow 
TABLE I. POWER CONSUMPTION OF CHARGE PUMP IN DIFFERENT CORNER CASES AT 200MHZ

\begin{tabular}{|c|c|}
\hline Corner case & Power consumption \\
\hline Typical_Typical & $357.6 \mu \mathrm{W}$ \\
\hline Fast_Fast & $511.1 \mu \mathrm{W}$ \\
\hline Fast_Slow & $346.1 \mu \mathrm{W}$ \\
\hline Slow_Fast & $159.1 \mu \mathrm{W}$ \\
\hline Slow_Slow & $169.0 \mu \mathrm{W}$ \\
\hline
\end{tabular}

\section{CONCLUSIONS}

The proposed circuit in this paper is a charge pump with the capability of being used in the PLL of the oscillator inside PMUs. This charge pump has a wide range of frequency and high voltage swing. The simulation results performed in ADS software and TSMC CMOS 0.18um technology, shows that this circuit only consumes $390 \mu \mathrm{W}$ power in supply voltage of $1.8 \mathrm{~V}$, and has a maximum current of $16.43 \mu \mathrm{A}$. The proposed charge pump is able to operate in a wide frequency range from $33 \mathrm{MHz}$ to $550 \mathrm{MHz}$ and generate output control voltage in the range of $0 \mathrm{~V}$ to $1.403 \mathrm{~V}$ at $50 \mathrm{MHz}$.

TABLE. II. COMPARISON OF PERFORMANCE OF PROPOSED CHARGE PUMP WITH PREVIOUS WORKS

\begin{tabular}{|c|c|c|c|c|c|c|}
\hline & {$[14]$} & {$[16]$} & {$[17]$} & {$[18]$} & {$[19]$} & This work \\
\hline Process CMOS & $0.18 \mathrm{um}$ & $0.18 \mathrm{um}$ & $90 \mathrm{~nm}$ & $0.18 \mathrm{um}$ & $90 \mathrm{~nm}$ & $0.18 \mathrm{um}$ \\
\hline Supply voltage & $1.2 \mathrm{~V}$ & $1.8 \mathrm{~V}$ & $1.2 \mathrm{~V}$ & $1.8 \mathrm{~V}$ & $1.2 \mathrm{~V}$ & $1.8 \mathrm{~V}$ \\
\hline Current of charge pump & $140 \mathrm{uA}$ & $100 \mathrm{uA}$ & $100 \mathrm{uA}$ & $475 \mathrm{uA}$ & N/A & $16.43 \mathrm{uA}$ \\
\hline
\end{tabular}

\section{REFERENCES}

[1] T. Becejac and P. Dehghanian, "PMU Multilevel End-to-End Testing to Assess Synchrophasor Measurements during Faults," in IEEE Power and Energy Technology Systems Journal, 2019. doi: 10.1109/JPETS.2019.2900064.

[2] N. Shams, P. Wall and V. Terzija, "Active Power Imbalance Detection, Size and Location Estimation Using Limited PMU Measurements," in IEEE Transactions on Power Systems, vol. 34, no. 2, pp. 1362-1372, March 2019.

[3] Shalini, S. R. Samantaray and A. Sharma, "Enhancing Performance of Wide-Area Back-Up Protection Scheme using PMU assisted Dynamic State Estimator," in IEEE Transactions on Smart Grid, 2018. doi: 10.1109/TSG.2018.2874946.

[4] J. Kitzig, S. Schlaghecke and G. Bumiller, "Power Quality Measurement System With PMU Functionality Based on Interpolated Sampling," in IEEE Transactions on Instrumentation and Measurement, 2018. doi: 10.1109/TIM.2018.2863458.

[5] Ikbal, A. L. I., Aftab, M. A., \& Hussain, S. S. (2016). Performance comparison of IEC 61850-90-5 and IEEE C37. 118.2 based wide area PMU communication networks. Journal of Modern Power Systems and Clean Energy, 4(3), 487-495.

[6] T. Nikas, E. Pikasis, A. Bogris and D. Syvridis, "A Microwave Optoelectronic PLL Synthesizer Based on Optical Comb Reference," in IEEE Photonics Technology Letters.

[7] M. Estebsari, M. Gholami, and M.J. Ghahramanpour, "A novel charge pump with low current for low-power delay-locked loops," Circuits, Systems, and Signal Processing, 36 (9), 3514-3526, 2017.

[8] M. Estebsari, M. Gholami and M. J. Ghahramanpour, "Overview of Analog Wide Range Delay Locked Loops," Recent Advances in Electrical \& Electronic Engineering (Formerly Recent Patents on Electrical \& Electronic Engineering), 11(4), 470-483, 2017.

[9] Y. You and J. Chen, "A Temperature-Compensated LC-VCO PLL and a Power-Efficient Decision Feedback Equalizer for MultiStandard Serial Links," 2018 14th IEEE International Conference on
Solid-State and Integrated Circuit Technology (ICSICT), Qingdao, 2018, pp. 1-4.

[10] M. Estebsari, M. Gholami, and M.J. Ghahramanpour, "A wide frequency range Delay Line for fast-locking and low power DelayLocked-Loops", Analog Integrated Circuits and Signal Processing, vol. 90, Issue 2, p.p. 427-434, February 2017.

[11] M. Estebsari, M. Gholami, and M.J. Ghahramanpour. "A wide range delay locked loop for low power and low jitter applications," International Journal of Circuit Theory and Applications, 46(3), 401-414, 2018.

[12] Y. Chen, P.-I. Mark and Y. Zhou, "Self-tracking charge pump for fast-locking PLL", Electronics letters, Vol.46, No. 11, p.p. 755-757, May 2010.

[13] Lee, J.S, Keel, M.S, Lim, S.I, and Kim, S, "Charge pump with perfect current matching characteristics in phase-locked-loops", Electronics letters, Vol.36, No.23, p.p.1907-1908, November 2000.

[14] Liu, P, Sun, P, Jung, J, and Heo, D, "PLL charge pump with adaptive body-bias compensation for minimum current variation", Electronics Letters, Vol.48, No.1, pp. 16-18, January 2012.

[15] N. Joram, R. Wolf and F. Ellinger, "High swing PLL charge pump with current mismatch reduction", Electronics Letters, vol. 50, no. 9, pp. 661-663, 2014.

[16] Sun Zheng, S., and Li, Z. "A novel CMOS charge pump with high performance for phase-locked loops synthesizer". IEEE Int. Conf. Communication Technology, China, 2011, pp. 1062-1065.

[17] Sheng Chen, Zhiqun Li and Qin Li, "An improved high speed charge pump in $90 \mathrm{~nm}$ CMOS technology," 2011 IEEE 13th International Conference on Communication Technology, Jinan, 2011, pp. 10951098.

[18] Hong, X., Zhiqun, L., Zhigong, W., Wei, L., \& Li, Z. (2007). A charge pump design for low-spur PLL.

[19] Suraj Kumar Saw, Payali Das, Madhusudan Maiti, Alak Majumder (2017). A 90nm Design of Charge Pump Circuit for Perfect Current Matching. 6th international conference on computing, communication and sensors networks, 2017. 